\title{
Mitosis-specific kinesins in Arabidopsis
}

\author{
Marleen Vanstraelen ${ }^{1}$, Dirk Inzé ${ }^{1}$ and Danny Geelen ${ }^{2}$
}

\author{
${ }^{1}$ Department of Plant Systems Biology, Flanders Interuniversity Institute for Biotechnology (VIB), Ghent University, \\ Technologiepark 927, B-9052 Gent, Belgium \\ ${ }^{2}$ Department of Plant Production, Faculty of Bioscience and Bio-engineering, Ghent University, Coupure links 653 , B-9000 Gent, \\ Belgium
}

\begin{abstract}
Kinesins are a class of microtubule-associated proteins that posses a motor domain for binding to microtubules and, in general, allows movement along microtubules. In animal mitosis, they function in spindle formation, chromosome movement and in cytokinesis. In addition to the spindle, plants develop a preprophase band and a phragmoplast that might require multiple kinesins for construction and functioning. Indeed, several kinesins play a role in phragmoplast and cell plate dynamics. Surprisingly few kinesins have been associated with the spindle and the preprophase band. Analysis of expression datasets from synchronized cell cultures indicate that at least 23 kinesins are in some way implicated in mitosis-related processes. In this review, the function of kinesins in animal and plant mitoses are compared, and the divergence that originates from plant-specific aspects is highlighted.
\end{abstract}

\section{Specialized kinesins for mitotic plant cells}

During cell division, microtubules (MTs) arrange into dynamic structures that drive the different stages of mitosis. The dynamic organization of MTs requires the functional cooperation of different MT-associated proteins (MAPs) [1]. Kinesins use a motor to convert the energy of ATP into movement along the MT or to control the (de)polymerization of MTs [2]. In animal cells, the role of different kinesins in distinct mitotic stages is well documented. Even though kinesins are strongly represented in plant genomes, with the exception of the class kinesin 2 and 3 for which there are no homologs, only a few members have been implicated in spindle organization, chromosome movement and cytokinesis [3]. Plants have additional, strongly diverged kinesins that do not classify within the subfamilies that have been defined to date [4]. These kinesins could be involved in the organization of the unique mitotic MT arrays, the preprophase band (PPB) and the phragmoplast that occur in plants but are absent in other eukaryotes [1]. The completion of sequencing the Arabidopsis thaliana genome and the classification of kinesins into different functional subfamilies [5,6], along with genome-wide expression analyses $[7,8]$, allows us to make predictions about kinesin functioning in dividing plant cells.

\section{Arabidopsis kinesins up-regulated during M-phase}

Progression through the cell cycle involves several hundreds of genes, which are regulated both at the

Corresponding author: Geelen, D. (danny.geelen@ugent.be).

Available online 10 March 2006 transcriptional and post-translational level. Transcript profiling of synchronized Arabidopsis tissue culture cells has identified 23 kinesins that are up-regulated during mitosis [7,8] (Figure 1, Tables 1 and 2). The promoter sequence of the mitosis activation motif (MSA) is a plantspecific $c i s$-acting element that drives periodic activation of gene expression at the start of mitosis or at the M phase of the cell cycle $[9,10]$. This cis element is strongly represented in the promoter sequences of the mitosis upregulated kinesins (Tables 1 and 2). The kinesin NACK1 of tobacco (Nicotiana tabacum) contains two MSA sequences in the promoter region that are responsible for mitosisdependent expression [11]. Accordingly, the NACK1 protein accumulates during M-phase, which is consistent with its function in cell plate expansion [12]. The MSA element is absent in the immediate upstream region of kinesins At4g21270, At4g05190 and At5g27550, suggesting that either the MSA sequence has degenerated beyond computer-assisted recognition or another mitosisspecific element exists for the expression of these particular kinesins.

\section{Cell cycle-controlled phosphorylation of kinesins}

Cyclin-dependent kinases (CDKs) regulate progression through the cell cycle by reversible phosphorylation of downstream effectors [13]. In animal cells, CDKA phosphorylation regulates the MT-binding activity of kinesin members of the Kinesin-4, Kinesin-5, Kinesin-6 and Kinesin-7 subfamilies that are required for spindle functioning during mitosis [14-16]. CDKA;1 phosphorylation sites are present in 14 out of the 23 kinesins that are mitotically up-regulated (Tables 1 and 2). The phosphorylation of Kinesin-5 (also known as BimC-type kinesins) Eg5 and KLP61F at a consensus CDKA phosphorylation site in the C-terminal BimC box is necessary to localize these proteins to the mitotic spindle in Xenopus laevis egg cells and Drosophila melanogaster embryos, respectively $[17,18]$. There are four Kinesin-5 homologs in Arabidopsis (i.e. AtKRP125a or At2g37420, AtKRP125b or At2g36200, AtKRP125c or At2g28620, and At3g45850) that, together with tobacco TKRP125, contain the conserved phosphorylation site embedded in their BimC box. AtKRP125a, AtKRP125b and AtKRP125c are up-regulated during mitosis whereas At3g45850 is not. By contrast, the Arabidopsis Kinesin-4 and Kinesin-7 family members lack a classic CDKA;1 phosphorylation site (Table 1), suggesting that unlike their animal counterparts, they are not regulated by CDK phosphorylation. In addition to control by CDK-type kinases, Aurora kinases have also 


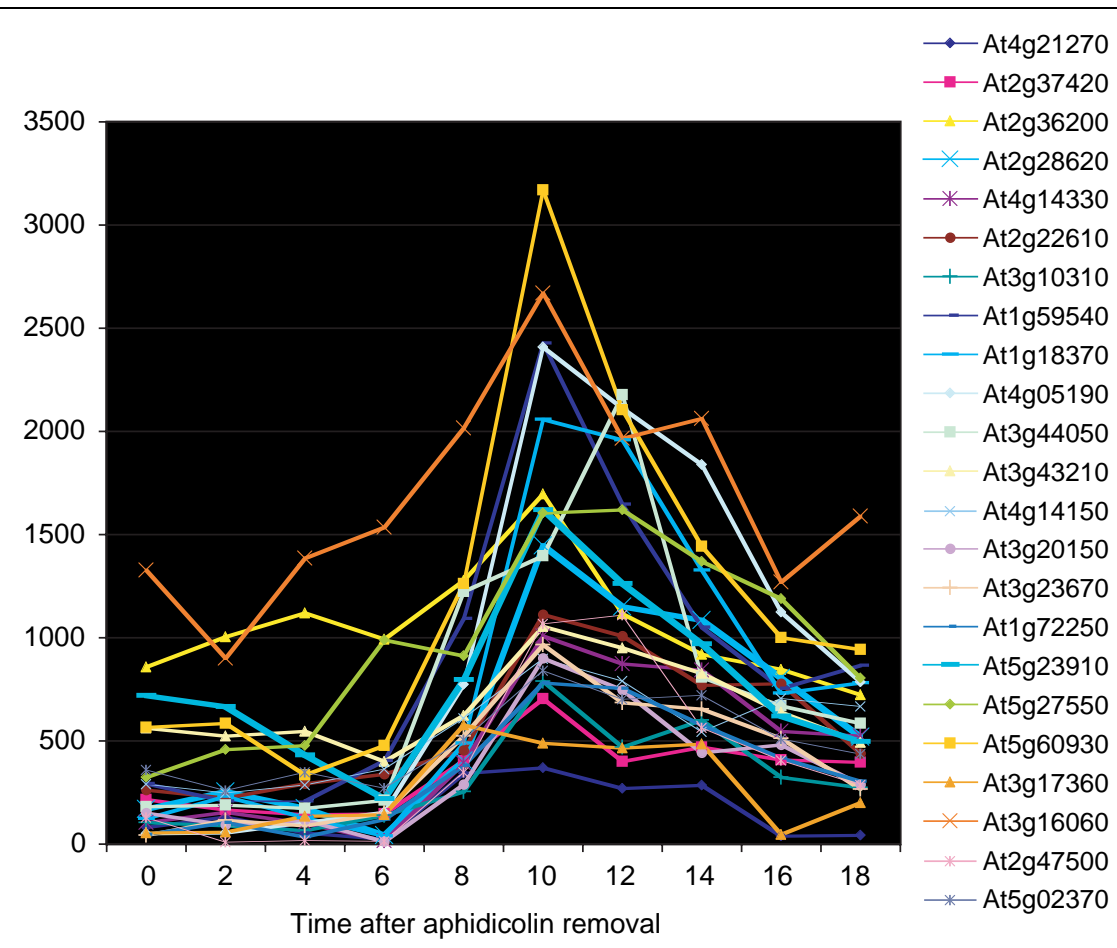

$\overline{\text { TRENDS in Plant Science }}$

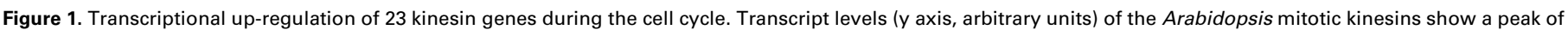
expression at mitosis, $10 \mathrm{~h}$ after aphidicolin release ( $\mathrm{x}$ axis, hours).

been shown to play important roles in the regulation of the subcellular localization of Kinesin-6 kinesins through phosphorylation. Aurora kinases are present in plants and localize to the spindle and cell plate [19]. Because Kinesin-6 kinesins are absent in Arabidopsis, it remains to be seen whether Aurora kinases have a role in kinesin regulation. The recent finding that Aurora $B$ also phosphorylates the Kinesin-5 BMK-1 in Caenorhabditis elegans has opened new avenues for investigation in plants [20]. Hitherto, there has been no direct evidence for CDK- or Aurora-dependent phosphorylation of kinesins. KCA, belonging to the Kinesin-14 group, has been analyzed in the most detail and has been shown to bind CDKA in two-hybrid assays and in vitro precipitation tests [21,22]. Furthermore, KCA is phosphorylated by an olomoucine-sensitive kinase, possibly a cell cycle-dependent kinase, in Sf9 insect cells [21]. Mutagenesis of two adjacent, putative CDK phosphorylation sites in the tail domain of KCA1 to glutamate codons, such that serine phosphorylation is mimicked, results in a failure to target the GFP-tagged protein correctly to the cell plate and the plasma membrane [23]. KCA-GFP with the same serines changed to non-phosphorable alanines accumulates in the cytoplasm, indicating that dephosphorylation is required for membrane localization. The KCA-related data are the first evidence for phosphorylation-controlled cellular distribution of kinesins in plants.

\section{Cell cycle control of the kinesin protein level}

For progression through the different steps of the mitotic phase, successive phosphorylation events are needed as well as the removal of proteins by proteolytic degradation. A well-studied event of collective removal of proteins takes place at anaphase during which key cell cycle regulators, such as cyclins and structural proteins, are rapidly degraded via a multistep process involving ubitiquitination and subsequent targeting to the $26 \mathrm{~S}$ proteasome. The targeting to the proteolytic complex relies on conserved domains, also known as destruction boxes, present in proteins that are targeted for proteolytic degradation [24]. The MAP Ase1 of yeast (Saccharomyces cerevisiae) carries a D-type destruction box that, when mutated, prevents proteolytic degradation and spindle disassembly [25]. A conserved D-box is also present in some members of the MAP65 protein family that show similarity to Ase1. For instance, the AtMAP65-4 disappears at the end of anaphase, suggesting that this protein might also be removed via the anaphase promoting complex (APC) [26]. One or more conserved D-boxes occur in 22 out of the 23 kinesins with mitosis-dependent expression (Tables 1 and 2). The strong conservation of the D-box supports a general role for protein degradation in controlling plant kinesin function. However, D-box-dependent or other proteolytic degradation of plant kinesins has not yet been demonstrated directly. The $26 \mathrm{~S}$ proteasome inhibitor MG132 arrests mitotic cells at metaphase because of the activation of the spindle checkpoint mechanism [27]. MG132 also blocks the M phase, G1 phase transition whereby it protects the TKRP125 from degradation and provokes the formation of extra phragmoplasts [28].

For several plant kinesins, protein levels have been investigated throughout the cell cycle in synchronized Bright Yellow-2 (BY-2) cells [12,29-31]. The protein levels 
Table 1. Mitotic kinesins in Arabidopsis belonging to defined kinesin families ${ }^{a}$

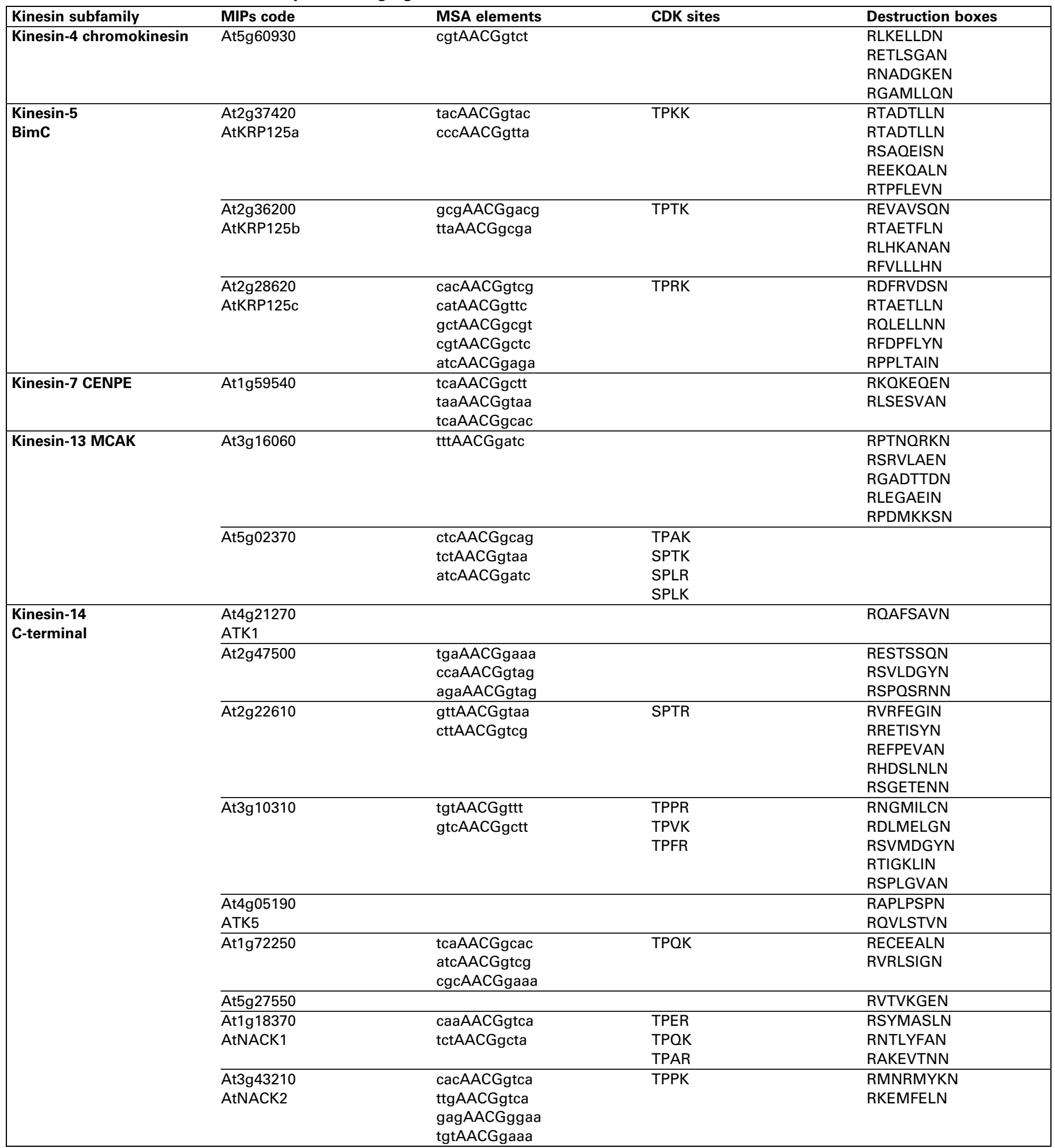

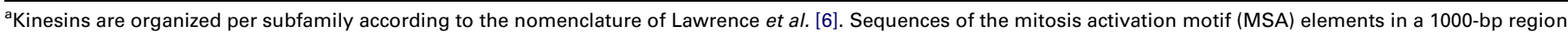
upstream of the ATG start codon, CDKA phosphorylation sites (S/T-P-x-K/R) and destruction boxes $\left(R-x_{2}-L-x_{4}-N\right)$ are shown.

of the kinesins ATK2, ATK3, TKRP125, NACK1 and KCBP of tobacco rise during M-phase and decline at mitotic exit. The Arabidopsis counterparts of ATK2, ATK3 and KCBP are not significantly up-regulated in micro arrays of synchronized cells $[7,8]$, suggesting that control over transcript and protein production is not a conserved mechanism in plant kinesin functioning.
M-phase-specific kinesins related to animal kinesins In animal cells, minus-end-directed kinesins are of major importance for the organization of the spindle poles (Figure 2). The founding member of this class, Ncd, localizes to the spindle poles and cooperates with dynein to bundle MTs that are attached to the centrosome at the poles [32]. As a general rule, kinesins with a motor domain 
Table 2. Mitotic kinesins specific to plants ${ }^{a}$

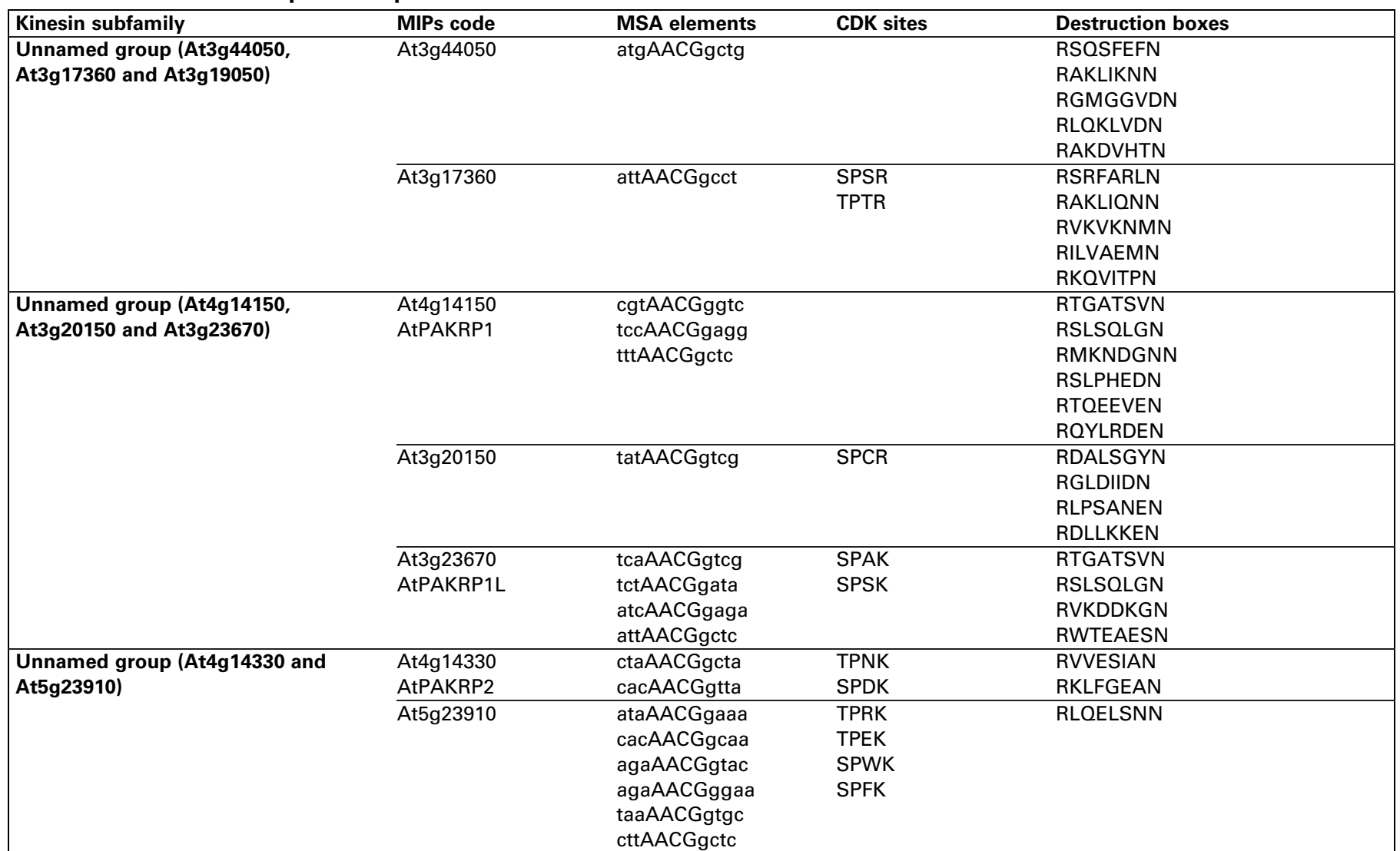

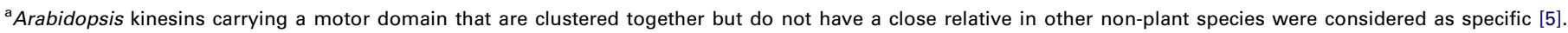

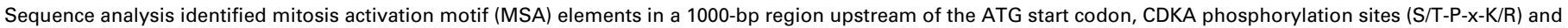
destruction boxes $\left(R-x_{2}-L-x_{4}-N\right)$.

near the C-terminal end exhibit minus-end directionality. The Arabidopsis genome contains $21 \mathrm{C}$-terminal kinesins now classified as Kinesin-14, of which seven are transcriptional and up-regulated at mitosis $[6,8]$. The Ncd-related ATK1 and ATK5 have been shown to move in the minusend direction of MTs and to associate with spindle MTs where they are required for focusing the MTs at the poles, which is similar to Ncd activity (Figure 2) [33-36]. Compared with other organisms, the Kinesin-14 group is relatively large. It has been postulated that these kinesins fulfill functions normally performed by cytoplasmic dynein [37]. However, some of the C-terminal kinesins have distinct properties by which they differentiate themselves from the dynein characteristics. For instance, MT-binding by kinesin KCBP is inhibited upon interaction with calcium-calmodulin [38-40]. Calcium levels rise during mitosis [41]. Yet, KCBP localizes to the spindle poles in Haemanthus endosperm, possibly through the interaction with other proteins that are components of the spindle pole complexes. Because of this localization at the poles, it has been proposed that KCBP bundles MT minus-ends in a similar way to Ncd (Figure 2) $[42,43]$.
Kinesin-5 members antagonize the forces of the Kinesin-14 subfamily members to establish spindle bipolarity [2]. Kinesin-5 KLP61F of Drosophila and Eg5 of human are plus-end-directed bipolar homotetramers that cross-link and slide antiparallel MTs of the spindle midzone, thereby generating poleward forces. Failure of Kinesin- 5 members to associate with the spindle results in the formation of monopolar spindles [17,44]. This phenotype can be partially rescued by mutations in the C-terminal kinesin Ncd, suggesting that a balance between forces directed towards plus-ends and minusends establishes the bipolar spindle [44]. A mechanism of balancing forces seems conserved because similar results were obtained in yeast and fungi $[45,46]$. Because the Arabidopsis AtKRP125 is predominantly concentrated at the spindle midzone, this Kinesin-5 probably acts in a similar way to BimC and antagonizes the force of the minus-end-directed kinesin ATK1 (Figure 2).

The plant spindle is formed in the absence of centrosomes. Consistently, the spindle poles are broader than those in animal cells [47]. Centrosomes are also absent in Drosophila oocytes [32,48]. Here, acentrosomal

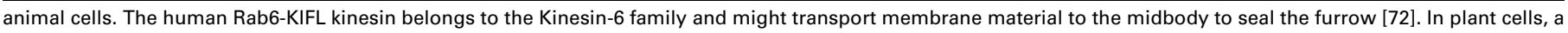
phragmoplast is built between the separated nuclei. This bipolar structure of MTs guides the transport of membrane vesicles to the division plane. Vesicle fusion results in the

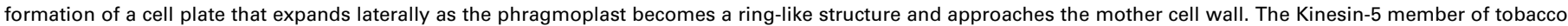

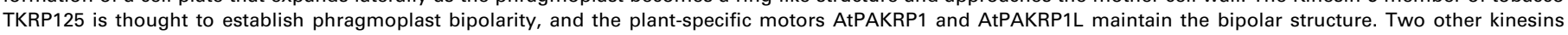
AtNACK1 and ATNACK2 function in the reorganization of MTs from a cylindrical structure into a ring-like structure [80]. The Kinesin-14 members KCA1 and KCA2 are putative

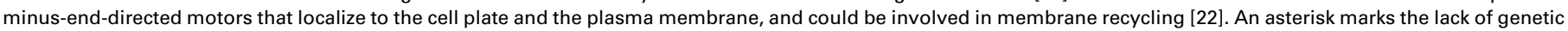
evidence for plant kinesin function. 
Plant

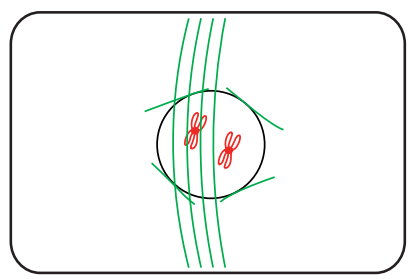

(a) Prophase
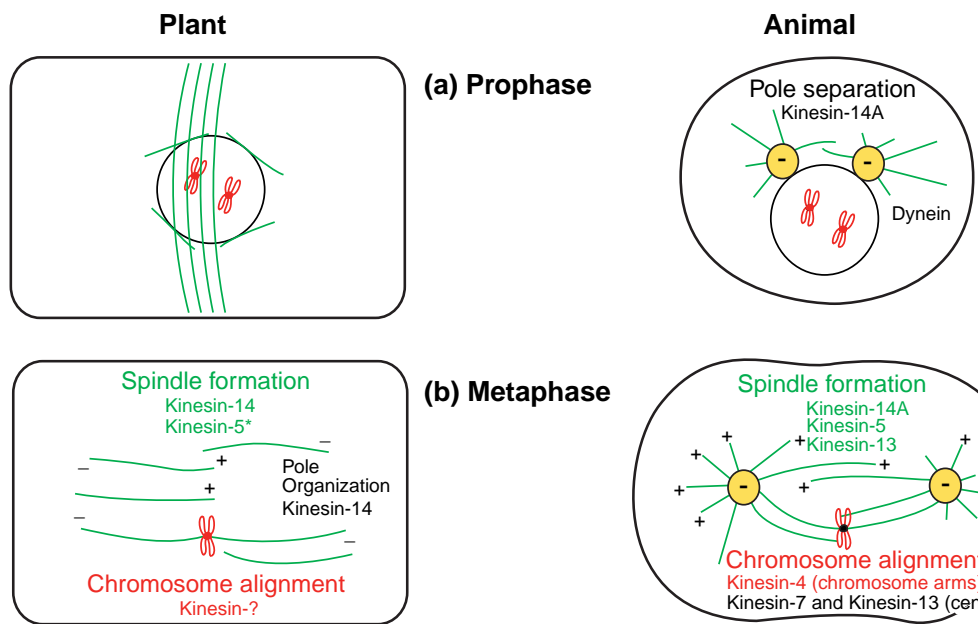

(b) Metaphase

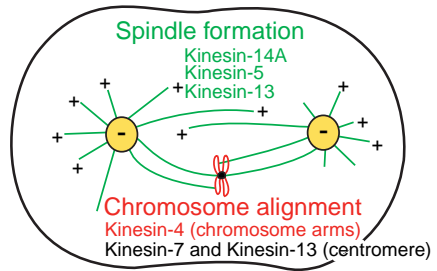

(c) Anaphase A

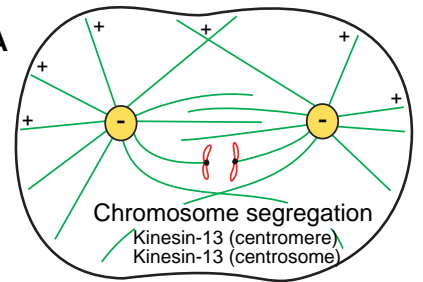

(d) Anaphase B
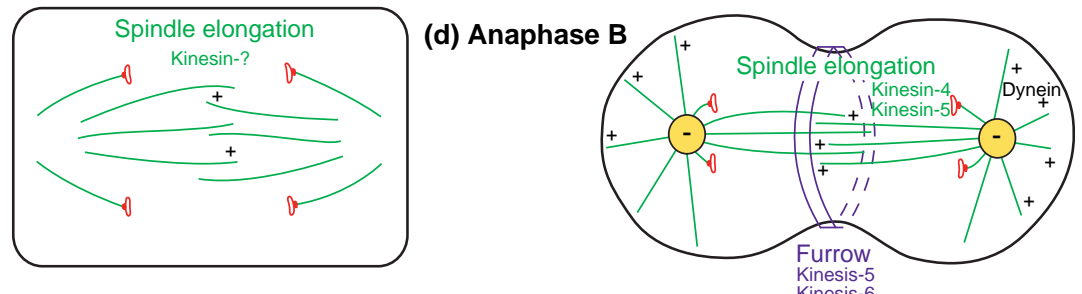

Phragmoplast formation Kinesin-5*

AtPAKRP $1 / 1 L^{*}$

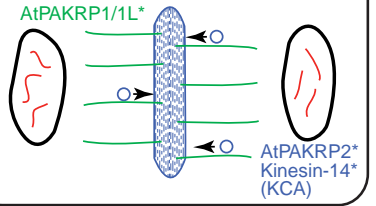

(e) Cytokinesis

Early

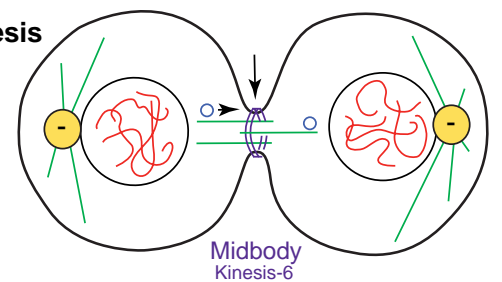

Late

$\overline{\text { TRENDS in Plant Science }}$

Figure 2. Kinesin motor proteins in plant and animal cell divisions. Overview of the different mitotic stages and the kinesin subfamilies that play a role in key events in mitotic progression. Many of the animal kinesins that are known to function in mitosis have been shown to be involved in multiple roles in different mitotic processes (see, for example, the Kinesin- 4 and the depolymerizing Kinesin-13 subfamily). (a) During prophase in animal cells, centrosomes migrate to opposite sides of the nucleus, thereby constructing the spindle. This migration is triggered by the outward pulling forces of dynein at the cell cortex and by the antagonistic forces of Kinesin-14A (Ncd) acting on non-kinetochore MTs [81]. In plant cells, the MTs of the PPB encircle the prophase spindle, which is built and nucleated at the nuclear surface. Motor proteins involved in PPB or prophase spindle formation have not been identified to date. (b) During metaphase, sister chromatids face opposite poles of the bipolar spindle. Spindle poles are broad in plant cells and poles are focused at the centrosomes in animal cells. Antagonistic forces of Kinesin-14, such as Ncd [81] and Arabidopsis ATK1 [35] and Kinesin-5, such as DmKLP61F [81], HsEg5 or AtTKRP125 establish spindle bipolarity, whereas Kinesin-14 members Ncd, ATK1 and ATK5 focus on the minus-ends of the spindle MTs [32-36]. Furthermore, members of the depolymerizing Kinesin-13 family (DmKLP10A and HsKIF2A) function in mitotic spindle assembly and maintenance [62]. Chromosomes are aligned by numerous kinesins in animal cells. Kinesin-4 members, such as Drosophila DmKLP3A or human and Xenopus KID, act on the chromosomal arms, whereas Kinesin-7 members (DmCENPmeta and HsCENP-E) and Kinesin-13 members (HsMCAK) function at the centromere [55-57,60,82]. (c) During anaphase A, chromosomes are separated to the spindle poles. The combined action of depolymerizing kinesins belonging to the Kinesin-13 family, which chew up MTs from the centromere (DmKLP59C and HsMCAK) and the centrosome (DmKLP10A or HsKIF2A), results in chromosome segregation [62]. (d) Later, during anaphase B, spindle poles are further separated. The Kinesin-5 motor DmKLP61F and dynein at the cortex drive spindle elongation, assisted by Kinesin-4 members. During late anaphase in animal cells, the cleavage furrow, which contains a contractile ring of actin-myosin, contracts and bundles the MTs into a central spindle. Both Kinesin-5 motors and Kinesin- 6 motors function in the organization of the central spindle [65]. (e) During cytokinesis, the cleavage furrow further ingresses, thereby concentrating the central spindle into a compact midbody in 
spindle assembly is accomplished in two steps: first, the spontaneous nucleation of MTs around condensed chromatin, followed by the sorting of randomly oriented MTs into a bipolar spindle involving the antagonizing action of Kinesin-5 and Kinesin-14 members [48]. The structural organization of the spindle MTs in plant cells starts before the nuclear envelope is broken down; therefore, it cannot rely directly on MT nucleation from chromatin. Nucleation seems to take place depending on $\gamma$-tubulin and Spc98p at the nuclear surface $[49,50]$. How spindle bipolarity is accomplished is not known. In the absence of ATK1, the spindle poles are wider than normal but still bipolar, indicating that minus-end-directed kinesins are needed merely to focus the MTs at the poles [34,36].

Plant Kinesin-5 members, such as TKRP125, not only associate with the spindle but also with the phragmoplast [30]. The phragmoplast MTs show a poleward translocation that is inhibited in the presence of TKRP125 antibody, in line with a role for TKRP125 in sliding MTs that are arranged in an antiparallel fashion. Recently, the tomographic analysis of phragmoplasts in whole-plant tissues of Arabidopsis has cast doubt on the commonly accepted idea that MTs interdigitate at the phragmoplast center [51]. If the MT plus ends are not overlapping, the activity of Kinesin-5 kinesins cannot operate as a result of the opposing phragmoplast MTs sliding. How then does the TKRP125 antibody inhibit outward translocation of phragmoplast MTs in permeabilized tobacco BY-2 cells? Perhaps the phragmoplasts in BY-2 cultured cells are organized differently to those in Arabidopsis and the MTs might temporally overlap, in a similar way to what occurs in miniphragmoplasts in endosperm tissue [52,53]. Alternatively, short stretches of MTs arranged in an antiparallel fashion occur near the plus-ends of phragmoplast MTs, which could be targeted by the TKRP125 kinesin. By pushing these short MTs against the centrally located cell plate via antiparallel sliding, the longer phragmoplast MTs would show an outward motion or a tread millingtype of translocation [30].

After nuclear envelope breakdown, pairs of sister chromatids associate with the spindle and oscillate until they are bi-oriented (attached to both spindle poles) and aligned at the metaphase plate [54]. For chromosome alignment, kinesins from different subfamilies must act cooperatively, generating poleward or antipoleward forces. Members of the Kinesin-4, Kinesin-7 and Kinesin-13 subfamily contribute to chromosome alignment [55-57]. Chromosome oscillations until metaphase alignment, which probably depend on the activity of kinesins, have been observed in plants [58].

Immunodepletion of Kinesin-4 Xkid, also known as chromokinesin, in frog egg extracts results in chromosome misalignment at metaphase [59]. Kinsin-4 members also function in chromosome condensation, segregation, spindle organization and cytokinesis as shown for Drosophila KLP3A (Figure 2) [60]. Arabidopsis has three kinesins with high levels of similarity to chromokinesins, one of which is up-regulated during mitosis (Table 1). The AtFRA1 homolog is not up-regulated during cell division and does not appear to be implicated in cell division. Indeed, its role is more consistent with the orientation of cellulose microfibrils in cell walls along with the MTs in the cortical array [61]. These findings underline the risk of drawing conclusions about the function of kinesins based on the extrapolation of amino acid conservation of the motor domain.

Kinesin-13 family members (also designated MCAK or Kin-I in mammals) depolymerize MTs and contribute to multiple aspects of chromosome movement [62]: they localize to the centromeres, centrosomes and spindle midzone during mitosis; depletion or disruption of MCAK activity has shown that they function in spindle maintenance, chromosome alignment and segregation; and they control the proper attachment of MTs to sister chromatids [57]. Three Kinesin-13 members are present in mammals (Kif2A, Kif2B and MCAK) and in Drosophila (Klp10A, Klp59C and Klp59D) [6]. MTs from the centrosome and the centromere are depolymerized by Kif2A and Klp10A and by MCAK and Klp59C, respectively (Figure 2). Arabidopsis contains two members of the Kinesin-13 subfamily, AtKinesin-13A and AtKinesin-13B [3]. AtKinesin-13B is controlled by mitosis and Atkinesin-13A is not, but they both lack a Lys-rich neck motif that is commonly found in other Kinesin-13 members, implying functional divergence from their animal counterparts [3]. Indeed, Atkinesin-13A localizes to Golgi stacks and contributes to Golgi distribution in Arabidopsis trichomes [63].

Members of the Kinesin-7 subfamily control the interaction between the MT plus-ends of the spindle and the kinetochores of the chromosomes, both during metaphase alignment and anaphase segregation (Figure 2) [55,64]. Two Kinesin-7 kinesins are present in the Arabidopsis genome, the expression of one of which is controlled by mitosis (Table 1) [6].

The homology-based survey indicates that kinesins with putative functions related to chromosome movements are represented in Arabidopsis as multiple copies, of which a subset is mitotically expressed. This does not exclude the constitutively active copies from involvement in chromosomal movement because transcript levels do not always reflect protein accumulation, and kinesin function might also be controlled at the protein level.

\section{M-phase-specific kinesins unique to plants}

Animal cytokinesis is accomplished by an actin-myosin ring emerging at the cell equator to recruit components necessary for ingressing the plasma membrane. As a result, interpolar MTs from the anaphase spindle are bundled into a central spindle that is further compressed into a compact midbody at the end of telophase (Figure 2) [65]. Plants construct a phragmoplast consisting of antiparallel MTs and perform cytokinesis without constriction. The MTs guide the Golgi-derived vesicles to the midline where they fuse to form a cell plate (Figure 2) [66]. Thus, animal cells depend on a mechanism requiring contraction whereas plant cells use a mechanism involving centrifugal expansion [65]. Despite this mechanistic difference, the central spindle in animal cells has properties similar to those of the phragmoplast in plant cells [67]. During furrow ingression, membrane vesicles accumulate at the leading edge of the cleavage furrow, which is 
blocked upon drug-stimulated MT depolymerization [68,69]. Furthermore, Brefeldin A, an inhibitor of vesicle traffic machinery, suppresses the terminal phase of cytokinesis in C. elegans and is a potent blocker of cell plate formation [70,71]. These findings support possible analogies in the roles that MT-based motor proteins play during animal and plant cytokinesis.

Human Kinesin-6 Rab6-KIFL, which is related to MKLP1, accumulates at the central spindle and becomes highly concentrated in the midbody where it might deliver vesicles from the Golgi apparatus to the site of membrane fusion [72,73]. Arabidopsis does not possess Kinesin-6 family members, suggesting that other kinesins execute the transport of Golgi-derived vesicles to the equator. Of the 29 kinesins that do not belong to any of the established kinesin subfamilies and are unique to plants [6], nine are up-regulated during mitosis and are probably implicated in plant-specific MT-related processes associated with the PPB or the phragmoplast. The plant-specific kinesins that are known to function in phragmoplast dynamics are indeed induced mitotically (Figure 1, Tables 1 and 2). AtPAKRP1 and the related AtPAKRP1L associate with the MTs of the cylinder-like and ring-like phragmoplasts and might maintain the bipolar structure of the phragmoplast once Kinesin-5 motors have established bipolarity $[74,75]$. Single mutants do not reveal any phenotype, perhaps because of mutual compensation for their respective loss of function [75]. AtPAKRP2, which is present in the Arabidopsis genome as a single gene, is not related to any other kinesin; it associates with the phragmoplast in a punctuate pattern and strongly accumulates at the cell plate in a Brefeldin A-sensitive manner [76]. Therefore, to date, AtPAKRP2 is the best candidate to deliver Golgi-derived vesicles to the phragmoplast midline. NACK1 (also named HIK [77]) and NACK2 (also named STUD [78] or TES [79]) play a role in phragmoplast MT dynamics [80]. NACK1 is essential for the completion of somatic cytokinesis and functions in the reorganization of MTs during the lateral expansion of the cell plate [12,77]; NACK2 is the closest homolog of NACK1 and takes part in the assembly of the radial MT array in male meiotic cytokinesis [78,79]. Both NACK1 and NACK2 interact with NPK1, a MAPKKK that controls phragmoplast expansion and cytokinesis [12]. Four mitotic up-regulated plant-specific kinesins that do not belong to the classic kinesin subfamilies are as yet uncharacterized (At3g44050, At3g17360, At3g20150 At5g23910; Table 2). Investigation of the function of these kinesins would be interesting to unravel plantspecific aspects of spindle and phragmoplast formation.

\section{Concluding remarks}

The combined effort of genome sequencing and genomewide transcription profiling in Arabidopsis has identified 23 mitotic kinesins that are categorized into different structural and functional subfamilies. These kinesins are the prime candidate motors to function in spindle organization and dynamics, chromosome movement, and cytokinesis. In addition to the transcriptionally activated kinesins, other kinesins contribute to mitosis without cell cycle-modulated RNA or protein levels. The scarceness of functional evidence for kinesins in spindle formation is striking. Indeed, most mitosis up-regulated kinesins have been assigned functions related to cytokinesis. No kinesins that have a role in chromosome movement have yet been identified and limited information is available on the role of kinesins in spindle dynamics. Sequence comparison suggests that the role of kinesins is only partially conserved in different aspects of chromosome alignment and segregation. Moreover, some homologs of animal kinesins with functions in chromosome movement are unrelated to plant cells and even seem to be important in processes that take place during interphase. Further functional divergence is expected because plant mitotic cytoskeletal structures display unique features. For instance, plants lack centrosomes and might use specially adapted kinesins to facilitate stabilization and condensation of MTs at the spindle poles. In addition, land plants form a PPB for which no counterpart exists in other eukaryotes. Until now, there is no evidence that kinesins contribute to the formation or dynamics of the PPB. Yet, in view of the significant number of plant-specific kinesins that have not been studied yet, they should be at the center of future research.

\section{Acknowledgements}

We thank Stephane Rombauts for bioinformatic support and Martine De Cock and Karel Spruyt for help in preparing the manuscript. M.V. is indebted to the Institute for the Promotion of Innovation by Science and Technology in Flanders for a predoctoral fellowship. D.G was a Postdoctoral Fellow of the Research Foundation-Flanders.

\section{References}

1 Lloyd, C. and Hussey, P. (2001) Microtubule-associated proteins in plants - why we need a map. Nat. Rev. Mol. Cell Biol. 2, 40-47

2 Sharp, D.J. et al. (2000) Microtubule motors in mitosis. Nature 407, 41-47

3 Lee, Y-R.J. and Liu, B. (2004) Cytoskeletal motors in Arabidopsis. Sixty-one kinesins and seventeen myosins. Plant Physiol. 136, 3877-3883

4 Miki, H. et al. (2005) Analysis of the kinesin superfamily: insights into structure and function. Trends Cell Biol. 15, 467-476

5 Reddy, A.S.N. and Day, I.S. (2001) Kinesins in the Arabidopsis genome: a comparative analysis among eukaryotes. BMC Genomics 2, 2

6 Lawrence, C.J. et al. (2004) A standardized kinesin nomenclature. J. Cell Biol. 167, 19-22

7 Menges, M. et al. (2003) Genome-wide gene expression in an Arabidopsis cell suspension. Plant Mol. Biol. 53, 423-442

8 Menges, M. et al. (2005) Global analysis of the core cell cycle regulators of Arabidopsis identifies novel genes, reveals multiple and highly specific profiles of expression and provides a coherent model for plant cell cycle control. Plant J. 41, 546-566

9 Ito, M. et al. (1998) A novel cis-acting element in promoters of plant B-type cyclin genes activates $\mathrm{M}$ phase-specific transcription. Plant Cell 10, 331-341

10 Araki, S. et al. (2004) Mitotic cyclins stimulate the activity of c-Myblike factors for transactivation of $\mathrm{G}_{2} / \mathrm{M}$ phase-specific genes in tobacco. J. Biol. Chem. 279, 32979-32988

11 Ito, M. et al. (2001) G2/M-phase-specific transcription during the plant cell cycle is mediated by c-Myb-like transcription factors. Plant Cell 13, 1891-1905

12 Nishihama, R. et al. (2002) Expansion of the cell plate in plant cytokinesis requires a kinesin-like protein/MAPKKK complex. Cell 109, 87-99 
13 Morgan, D.O. (1997) Cyclin-dependent kinases: engines, clocks, and microprocessors. Annu. Rev. Cell Dev. Biol. 13, 261-291

14 Reilein, A.R. et al. (2001) Regulation of molecular motor proteins. Int. Rev. Cytol. 204, 179-238

15 Ohsugi, M. et al. (2003) Cdc2-mediated phosphorylation of Kid controls its distribution to spindle and chromosomes. EMBO J. 22, 2091-2103

16 Goshima, G. and Vale, R.D. (2005) Cell cycle-dependent dynamics and regulation of mitotic kinesins in Drosophila $\mathrm{S} 2$ cells. Mol. Biol. Cell 16, 3896-3907

17 Blangy, A. et al. (1995) Phosphorylation by $\mathrm{p} 34^{c d c 2}$ regulates spindle association of human Eg5, a kinesin-related motor essential for bipolar spindle formation in vivo. Cell 83, 1159-1169

18 Sharp, D.J. et al. (1999) The bipolar kinesin, KLP61F, cross-links microtubules within interpolar microtubule bundles of Drosophila embryonic mitotic spindles. J. Cell Biol. 144, 125-138

19 Demidov, D. et al. (2005) Identification and dynamics of two classes of Aurora-like kinases in Arabidopsis and other plants. Plant Cell 17, 836-848

20 Bishop, J.D. et al. (2005) The Caenorhabditis elegans Aurora B kinase AIR-2 phosphorylates and is required for the localization of a BimC kinesin to meiotic and mitotic spindles. Mol. Biol. Cell 16, 742-756

21 Kong, L-J. and Hanley-Bowdoin, L. (2002) A geminivirus replication protein interacts with a protein kinase and a motor protein that display different expression patterns during plant development and infection. Plant Cell 14, 1817-1832

22 Vanstraelen, M. et al. (2004) A plant-specific subclass of C-terminal kinesins contains a conserved A-type cyclin-dependent kinase site implicated in folding and dimerization. Plant Physiol. 135, 1417-1429

23 Vanstraelen, M. et al. (2006) Cell cycle-dependent targeting of a Kinesin at the plasma membrane demarcates the division site in plant cells. Curr. Biol. 16, 308-314

24 Glotzer, M. et al. (1991) Cyclin is degraded by the ubiquitin pathway. Nature $349,132-138$

25 Juang, Y-L. et al. (1997) APC-mediated proteolysis of Ase1 and the morphogenesis of the mitotic spindle. Science 275, 1311-1314

26 Van Damme, D. et al. (2004) In vivo dynamics and differential microtubule-binding activities of MAP65 proteins. Plant Physiol. 136, 3956-3967

27 Genschik, P. et al. (1998) Cell cycle-dependent proteolysis in plants: identification of the destruction box pathway and metaphase arrest produced by the protease inhibitor MG132. Plant Cell 10, 2063-2075

28 Oka, M. et al. (2004) Inhibition of proteasome by MG-132 treatment causes extra phragmoplast formation and cortical microtubule disorganization during M/G1 transition in synchronized tobacco cells. Plant Cell Physiol. 45, 1623-1632

29 Mitsui, H. et al. (1996) Cell cycle-dependent accumulation of a kinesinlike protein, KatB/C in synchronized tobacco BY-2 cells. Plant Mol. Biol. 30, 177-181

30 Asada, T. et al. (1997) TKRP125, a kinesin-related protein involved in the centrosome-independent organization of the cytokinetic apparatus in tobacco BY-2 cells. J. Cell Sci. 110, 179-189

31 Bowser, J. and Reddy, A.S. (1997) Localization of a kinesin-like calmodulin-binding protein in dividing cells of Arabidopsis and tobacco. Plant J. 12, 1429-1437

32 Merdes, A. and Cleveland, D.W. (1997) Pathways of spindle pole formation: different mechanisms; conserved components. J. Cell Biol. 138, 953-956

33 Marcus, A.I. et al. (2002) Arabidopsis thaliana protein, ATK1, is a minus-end directed kinesin that exhibits non-processive movement. Cell Motil. Cytoskeleton 52, 144-150

34 Marcus, A.I. et al. (2003) A kinesin mutant with an atypical bipolar spindle undergoes normal mitosis. Mol. Biol. Cell 14, 1717-1726

35 Chen, C. et al. (2002) The Arabidopsis ATK1 gene is required for spindle morphogenesis in male meiosis. Development 129, 2401-2409

36 Ambrose, J.C. et al. (2005) A minus-end-directed kinesin with plusend tracking protein activity is involved in spindle morphogenesis. Mol. Biol. Cell 16, 1584-1592

37 Lawrence, C.J. et al. (2001) Dyneins have run their course in plant lineage. Traffic 2, 362-363

38 Narasimhulu, S.B. et al. (1997) Interaction of Arabidopsis kinesin-like calmodulin-binding protein with tubulin subunits: modulation by $\mathrm{Ca}^{2+}$-calmodulin. Plant J. 12, 1139-1149
39 Song, H. et al. (1997) In vitro motility of AtKCBP, a calmodulinbinding kinesin protein of Arabidopsis. Proc. Natl. Acad. Sci. U. S. A. 94, 322-327

40 Deavours, B.E. et al. (1998) $\mathrm{Ca}^{2+} /$ calmodulin regulation of the Arabidopsis kinesin-like calmodulin-binding protein. Cell Motil. Cytoskeleton 40, 408-416

41 Hepler, P.K. et al. (1993) Cytoskeletal dynamics in living plant cells. Cell Biol. Int. 17, 127-142

42 Smirnova, E.A. et al. (1998) Minus end-directed kinesin-like motor protein, Kcbp, localizes to anaphase spindle poles in Haemanthus endosperm. Cell Motil. Cytoskeleton 41, 271-280

43 Vos, J.W. et al. (2000) The kinesin-like calmodulin binding protein is differentially involved in cell division. Plant Cell 12, 979-990

44 Wilson, P.G. et al. (2004) Novel nuclear defects in KLP61F-deficient mutants in Drosophila are partially suppressed by loss of Ncd function. J. Cell Sci. 117, 4921-4933

45 Saunders, W.S. and Hoyt, M.A. (1992) Kinesin-related proteins required for structural integrity of the mitotic spindle. Cell 70, 451-458

46 O'Connell, M.J. et al. (1993) Suppression of the bimC4 mitotic spindle defect by deletion of $k l p A$, a gene encoding a KAR3-related kinesinlike protein in Aspergillus nidulans. J. Cell Biol. 120, 153-162

47 Wasteneys, G.O. (2002) Microtubule organization in the green kingdom: chaos or self-order? J. Cell Sci. 115, 1345-1354

48 Manandhar, G. et al. (2005) Centrosome reduction during gametogenesis and its significance. Biol. Reprod. 72, 2-13

49 Binarová, P. et al. (1998) Association of $\gamma$-tubulin with kinetochore/centromeric region of plant chromosomes. Plant J. 14, 751-757

50 Erhardt, M. et al. (2002) The plant Spc98p homologue colocalizes with $\gamma$-tubulin at microtubule nucleation sites and is required for microtubule nucleation. J. Cell Sci. 115, 2423-2431

51 Austin, J.R., II. et al. (2005) Quantitative analysis of changes in spatial distribution and plus-end geometry of microtubules involved in plant-cell cytokinesis. J. Cell Sci. 118, 3895-3903

52 Euteneuer, U. and McIntosh, J.R. (1980) Polarity of midbody and phragmoplast microtubules. J. Cell Biol. 87, 509-515

53 Otegui, M.S. et al. (2001) Three-dimensional analysis of syncytial-type cell plates during endosperm cellularization visualized by high resolution electron tomography. Plant Cell 13, 2033-2051

54 Murray, A.W. and Mitchison, T.J. (1994) Kinetochores pass the IQ test. Curr. Biol. 4, 38-41

55 Schaar, B.T. et al. (1997) CENP-E function at kinetochores is essential for chromosome alignment. J. Cell Biol. 139, 1373-1382

56 Antonio, C. et al. (2000) Xkid, a chromokinesin required for chromosome alignment on the metaphase plate. Cell 102, 425-435

57 Kline-Smith, S.L. et al. (2004) Depletion of centromeric MCAK leads to chromosome congression and segregation defects due to improper kinetochore attachments. Mol. Biol. Cell 15, 1146-1159

$58 \mathrm{Yu}, \mathrm{H}-\mathrm{G}$. et al. (1997) Neocentromere-mediated chromosome movement in maize. J. Cell Biol. 139, 831-840

59 Castro, A. et al. (2003) Xkid is degraded in a D-box, KEN-box, and A-box-independent pathway. Mol. Cell. Biol. 23, 4126-4138

60 Mazumdar, M. and Misteli, T. (2005) Chromokinesins: multitalented players in mitosis. Trends Cell Biol. 15, 349-355

61 Zhong, R. et al. (2002) A kinesin-like protein is essential for oriented deposition of cellulose microfibrils and cell wall strength. Plant Cell 14, 3101-3117

62 Moore, A. and Wordeman, L. (2004) The mechanism, function and regulation of depolymerizing kinesins during mitosis. Trends Cell Biol. 14, 537-546

$63 \mathrm{Lu}$, L. et al. (2004) An internal motor kinesin is associated with the Golgi apparatus and plays a role in trichome morphogenesis in Arabidopsis. Mol. Biol. Cell 16, 811-823

64 McEwen, B.F. et al. (2001) CENP-E is essential for reliable bioriented spindle attachment, but chromosome alignment can be achieved via redundant mechanisms in mammalian cells. Mol. Biol. Cell 12, $2776-2789$

65 Glotzer, M. (2001) Animal cell cytokinesis. Annu. Rev. Cell Dev. Biol. $17,351-386$

66 Verma, D.P.S. (2001) Cytokinesis and building of the cell plate in plants. Annu. Rev. Plant Physiol. Plant Mol. Biol. 52, 751-784

67 Bowerman, B. and Severson, A.F. (1999) Plant-like properties of animal cell cytokinesis. Curr. Biol. 9, R658-R660 
68 Bluemink, J.G. and Laat, S.D. (1973) New membrane formation during cytokinesis in normal and cytochalasin B-treated eggs of Xenopus laevis. I. Electron microscope observations. J. Cell Biol. 59, 89-108

69 Danilchik, M.V. et al. (1998) Requirement for microtubules in new membrane formation during cytokinesis of Xenopus embryos. Dev. Biol. 194, 47-60

70 Yasuhara, H. et al. (1995) Effects of Brefeldin A on the formation of the cell plate in tobacco BY-2 cells. Eur. J. Cell Biol. 66, 274-281

71 Skop, A.R. et al. (2001) Completion of cytokinesis in C. elegans requires a Brefeldin A-sensitive membrane accumulation at the cleavage furrow apex. Curr. Biol. 11, 735-746

72 Hill, E. et al. (2000) The Rab6-binding kinesin, Rab6-KIFL, is required for cytokinesis. $E M B O$ J. 19, 5711-5719

73 Fontijn, R.D. et al. (2001) The human kinesin-like protein RB6K is under tight cell cycle control and is essential for cytokinesis. Mol. Cell. Biol. 21, 2944-2955

74 Lee, Y-R.J. and Liu, B. (2000) Identification of a phragmoplast-associated kinesin-related protein in higher plants. Curr. Biol. 10, 797-800

75 Pan, R. et al. (2004) Localization of two homologous Arabidopsis kinesin-related proteins in the phragmoplast. Planta 220, 156-164
76 Lee, Y-R.J. et al. (2001) A novel plant kinesin-related protein specifically associates with the phragmoplast organelles. Plant Cell 13, 2427-2439

77 Strompen, G. et al. (2002) The Arabidopsis HINKEL gene encodes a kinesin-related protein involved in cytokinesis and is expressed in a cell cycle-dependent manner. Curr. Biol. 12, 153-158

78 Hülskamp, M. et al. (1997) The $S T U D$ gene is required for malespecific cytokinesis after telophase II of meiosis in Arabidopsis thaliana. Dev. Biol. 187, 114-124

79 Yang, C-Y. et al. (2003) TETRASPORE encodes a kinesin required for male meiotic cytokinesis in Arabidopsis. Plant J. 34, 229-240

80 Tanaka, H. et al. (2004) The AtNACK1/HINKEL and STUD/ TETRASPORE/AtNACK2 genes, which encode functionally redundant kinesins, are essential for cytokinesis in Arabidopsis. Genes Cells 9, 1199-1211

81 Kwon, M. and Scholey, J.M. (2004) Spindle mechanics and dynamics during mitosis in Drosophila. Trends Cell Biol. 14, 194-205

82 Yucel, J.K. et al. (2000) CENP-meta, an essential kinetochore kinesin required for the maintenance of metaphase chromosome alignment in Drosophila. J. Cell Biol. 150, 1-11

\section{Plant Science meetings in July 2006}

\section{Plant Growth Regulation Society of America (PGRSA) 33rd Annual Conference 9-12 July 2006 \\ Quebec City Hilton, Quebec City, Canada \\ http://www.griffin.peachnet.edu/pgrsa}

17th International Symposium on Plant Lipids

16-21 July 2006

East Lansing, MI, USA

http://www.ispl2006.msu.edu/index.html

XV FESPB 2006: Plants, People, Ecosystems and Applications

17-21 July 2006

Lyon, France

http://www.fespb2006.org/

5th International Conference on Mycorrhiza

23-27 July 2006

Granada, Spain

http://www.eez.csic.es/icom5/

Botany 2006

28 July - 3 August 2006

Chico, CA, USA

http://www.2006.botanyconference.org/

American Phytopathological Society

29 July - 2 August 2006

Québec, Canada

http://meeting.apsnet.org/ 